\title{
ADAPTACIÓN Y SELECCIÓN DE MAÍCES NATIVOS EN LA REGIÓN DEL BAJÍO DE MÉXICO POR SU CARACTERIZACIÓN MORFOLÓGICA
}

\author{
ADAPTATION AND SELECTION OF NATIVE MAIZE IN THE MEXICAN BAJIO \\ REGION BASED ON MORPHOLOGICAL CHARACTERIZATION
}

\section{J. Antonio Rangel-Lucio', Amalio Santacruz-Varela², Leobigildo Córdova-Téllez², J. Guadalupe García-Rodríguez ${ }^{3 *}$, Francisco Cervantes-Ortíz ${ }^{3}$, Humberto Vaquera-Huerta ${ }^{2}$ y Jesús Alberto Cuenca-Salgado ${ }^{3}$}

\begin{abstract}
'Tecnológico Nacional de México (TecNM), Instituto Tecnológico de Ciudad Victoria, Ciudad Victoria, Tamaulipas, México. ${ }^{2}$ Colegio de Postgraduados, Campus Montecillo, Programa de Recursos Genéticos y Productividad, Montecillo, Texcoco, Estado de México, México. ${ }^{3}$ TecNM, Instituto Tecnológico de Roque, Celaya, Guanajuato, México.
\end{abstract}

\section{RESUMEN}

La caracterización morfológica es un proceso de gran importancia para incorporar germoplasma de valor en los programas de introducción de maíz (Zea mays L.) con fines de mejoramiento genético. El objetivo del presente estudio fue caracterizar un grupo de 90 accesiones de 12 razas de maíz nativo de México: Bofo, Celaya, Coscomatepec, Elotes Occidentales, Harinoso de Ocho, Dulce de Jalisco, Mushito, Pepitilla, Serrano de Jalisco, Tablilla de Ocho, Tabloncillo y Zamorano Amarillo para identificar aquellas con mejores características productivas y de adaptación en el Bajío de México. Se utilizó un diseño de bloques completos al azar y se evaluaron caracteres agronómicos de la planta, espiga, mazorca y grano. Hubo diferencias significativas entre las accesiones de maíz nativo. El rendimiento de grano más alto se registró en las accesiones Gto-101 (8.3 $\left.\mathrm{t} \mathrm{ha}^{-1}\right)$, Dgo-18 y Jal-554 de la raza Celaya y Jal-633 $\left(6.0 \mathrm{t} \mathrm{ha}^{-1}\right)$ de Elotes Occidentales. La mayor altura de planta y de mazorca y dimensiones de la espiga (longitud de pedúnculo, del tramo del eje con ramas y del eje principal) se prentaron en las accesiones Ver-406 (raza Coscomatepec), Nay-214 (Harinoso de Ocho), Dgo-218 (Celaya), y en Nay-191, Nay-196 y Nay-217 (Bofo). El análisis de conglomerados integró a las razas Bofo, Celaya y Elotes Occidentales en los Grupos I, II y III, mientras que el Grupo IV fue integrado por Mushito, Pepitilla, Bofo y Tabloncillo, y el Grupo V por mezclas de las razas evaluadas, excepto Celaya. Fue posible identificar accesiones de las razas de maíz Bofo, Celaya y Elotes Occidenatles prometedoras para el Bajío de México.

Palabras clave: Zea mays, accesión, caracter agronómico, maíz nativo, producción de grano, razas de maíz mexicano.

\section{SUMMARY}

Morphological characterization is a process of great importance to incorporate valuable germplasm in the introduction programs of maize (Zea mays L.) for breeding purposes. The objective of this study was to characterize a group of 90 accessions of 12 maize races native to Mexico: Bofo, Celaya, Coscomatepec, Elotes Occidentales, Harinoso de Ocho, Dulce de Jalisco, Mushito, Pepitilla, Serrano de Jalisco, Tablilla de Ocho, Tabloncillo and Zamorano Amarillo to identify those with the best productive characteristics and adaptation to the Bajio region in Mexico. A randomized complete block design was used and agronomic traits of the plant, tassel, ear and grain were evaluated. There were significant differences between native maize accessions. The highest grain yield was registered in the accessions Gto-101 (8.3 $\left.\mathrm{t} \mathrm{ha}^{-1}\right)$, Dgo-18 and Jal-554 of the Celaya race and Jal-633 (6.0 $\left.\mathrm{t} \mathrm{ha}^{-1}\right)$ of Elotes Occidentales. The largest plant and ear height and tassel dimensions (length of the peduncle, of the branched section and the main axis) occurred in accessions Ver-406 (Coscomatepec race), Nay-214 (Harinoso de Ocho), Dgo-218 (Celaya), Nay-191, Nay-196 and Nay-217 (Bofo). The cluster analysis incorporated the Bofo, Celaya and Elotes Occidentales races into Groups I, II and III, while Group IV was integrated by Mushito, Pepitilla, Bofo and Tabloncillo, and Group V by mixture of the races evaluated, except Celaya. It was possible to identify promising accessions of Bofo, Celaya and Elotes Occidenatles maize races for the Mexican Bajio.

Index words: Zea mays, accession, agronomic character, grain production, Mexican maize races, native maize.

\section{INTRODUCCIÓN}

La distribución, el nivel de producción y consumo de grano y forraje y sus derivados, colocan al maíz (Zea mays L.) como cultivo de importancia mundial. La mayor diversidad genética de maíz se concentra en el sur de México y Guatemala (Coutiño-Estrada et al., 2010). Existe un registro de 64 razas de maíz reportadas en México (CONABIO, 2020), pero el manejo, migración de polen, selección e intercambio de semilla entre productores producen cambios y con frecuencia reducen la diversidad (Guillén-de la Cruz et al., 2014); es decir, la diversidad del maíz depende de factores biológicos, ambientales y antropocéntricos (Ortega-Villegas et al., 2018) y se estudia con varios propósitos, como conocer la variabilidad y plantear la clasificación en razas, contar con germoplasma suficiente y ampliar la variabilidad genética para mejoramiento (CONABIO-TEEB, 2017), lo cual se apoya en la caracterización y evaluación de los maíces nativos.

En el centro del país, la diversidad de poblaciones de maíz es dinámica y compleja; por ejemplo, al oriente de la Ciudad de México, a pesar del predominio de la raza Chalqueño es posible localizar a Arrocillo Amarillo, Ancho y Cacahuacintle (Herrera-Cabrera et al., 2004), y dicha complejidad se extiende hacia Puebla (Hortelano et 
al., 2012), donde Chalqueño y su variante Chalqueño Crema resultan comunes. Hacia el sur, en el Istmo de Tehuantepec, Oaxaca, predomina Zapalote Chico (CabreraToledo et al., 2019) con seis grupos, clasificados a su vez en tipos conocidos localmente como maíz chico y maíz grande (López et al., 2005), en tanto que en el municipio de Tlaxiaco en la región Mixteca Alta ocurre una variación morfológica y rendimiento importantes y las mazorcas guardan parecido con las razas Bolita, Serrano Mixe y Ancho (Chávez-Servia et al., 2011). Resultados de DiegoFlores et al. (2012) en la Mixteca Baja de Oaxaca indican que la espiga, mazorca y grano de 60 poblaciones fueron similares a los de las razas Celaya, Bolita, Pepitilla y Ancho; ciertos complejos raciales estuvieron asociados con la geografía y elevación. El análisis ecogeográfico determina el grado de adaptación climática de razas y accesiones para comprender la diversidad genética, planeación de colectas futuras y fundamentar programas de conservación in situ de maíz (Ruiz et al., 2008).

El concepto de raza de maíz ha evolucionado; Ron et al. (2006) describen a la raza como grupos de poblaciones con características numerosas y genes comúnes, que las agrupa y diferencía de otras, hereda sus características a generaciones futuras y ocupa un área ecológica particular. Muñoz (2005) agrega el individuo como componente básico del concepto de raza, el cual emparenta con genotipos similares. Esa evolución ha permitido la continuidad y ejecución de ensayos de caracterización de maíz, no sólo como parte de programas de mejoramiento sino también como fuente de materiales sobresalientes, factibles de producir en situaciones climáticas y edáficas desfavorables. La precipitación pluvial es escasa y errática en casi todo México, lo que también provoca producción baja de grano de maíz, la cual es estimada en $2.4 \mathrm{t} \mathrm{ha}^{-1}$ en temporal (SIAP, 2020). También se suman aspectos socioeconómicos contrastantes, como la tecnología agrícola destacada en la región Bajío de México, que emplea maíces mejorados y la escasa atención hacia maíces nativos. En este contexto, este estudio tuvo por objetivo caracterizar morfológicamente 90 accesiones de maíz nativo como prueba de su respuesta adaptativa, a fin de identificar y proponer materiales genéticos promisorios para reforzar la base genética en la región Bajío de México.

\section{MATERIALES Y MÉTODOS}

\section{Sitio experimental}

La evaluación de los materiales se realizó en Celaya, Guanajuato, México en un sitio con coordenadas $20^{\circ} 30^{\prime}$ 28" LN y 100 50' 00" LO y 1745 msnm. El clima de la región es BS ${ }_{1}$ hw(w)(e), con precipitación pluvial media anual de $599.2 \mathrm{~mm}$ principalmente en verano y temperatura media anual de $19.6^{\circ} \mathrm{C}$ (García, 1988). El suelo es Vertisol Pélico.

\section{Material genético}

El material genético evaluado consistió en 90 accesiones correspondientes a 12 razas de maíz y fue proporcionado por el Colegio de Postgraduados, Campus Montecillo, Estado de México, a través del proyecto "El Maíz Mexicano y su Diversidad Molecular". Las razas (y número de accesiones) fueron: Bofo (11), Celaya (17), Coscomatepec (1), Elotes Occidentales (16), Harinoso de Ocho (1), Dulce de Jalisco (6), Mushito (10), Pepitilla (10), Serrano de Jalisco (2), Tablilla de Ocho (7), Tabloncillo (8) y Zamorano Amarillo (1), con adaptación a altitudes intermedias.

\section{Siembra y manejo}

La siembra fue efectuada el 11 de mayo de 2009 bajo "punta de riego" (con un riego inicial), precipitación pluvial de $461 \mathrm{~mm}$ y dos riegos de auxilio durante el ciclo. La siembra fue de dos semillas por mata en forma manual cada $50 \mathrm{~cm}$, en surcos de $0.80 \mathrm{~m}$ de separación y $5.0 \mathrm{~m}$ de longitud, para una densidad de población de 48,000 plantas por hectárea. El manejo técnico del cultivo se basó en la recomendación de SAGARPA-INIFAP-SENASICA (2015). En el suelo se aplicó la dosis de fertilización 240-40-00 con urea y super fosfato de calcio triple, complementada con $2 \mathrm{~L} \mathrm{ha}^{-1}$ de Plantafol ${ }^{\circledR}$ (20-20-20 y micronutrimentos), cuando la planta alcanzó entre 0.40 y $0.60 \mathrm{~m}$ de altura. Las malezas se controlaron manual y mecánicamente (45 días después de la siembra), y con agroquímicos recomedados ( $1 \mathrm{~L} \mathrm{ha}^{-1}$ de 2,4-D amina en etapa vegetativa y $1 \mathrm{~L} \mathrm{ha-1}$ de Nicosulfuron en la fase reproductiva y maduración de grano).

\section{Diseño y unidad experimental}

Se utilizó un diseño experimental de bloques completos al azar con tres repeticiones. La unidad experimental fue de dos surcos de $5.0 \mathrm{~m}$ de longitud y $0.8 \mathrm{~m}$ de separación con dos plantas cada $0.50 \mathrm{~m}$.

\section{Variables medidas}

Las variables se y registraron conforme a la guía técnica para maíz (SAGARPA-SNICS, 2014). Los caracteres considerados fueron: a) agronómicos (producción de mazorca $(\mathrm{Rm})$ en $\mathrm{t}$ ha-1, producción de grano $(\mathrm{Rg})$ en $\mathrm{t} \mathrm{ha}^{-1}$, producción de olote (Ro) en tha-1 ${ }^{-1}$ días a floración masculina $(\mathrm{Fm})$, altura de planta (Ap) en $\mathrm{cm}$, altura de mazorca (Am) en cm y número de hojas arriba de la mazorca (Hm)]; b) de la espiga [longitud del pedúnculo ( $L p)$ en $\mathrm{cm}$, longitud del tramo del eje con ramas (Le) en cm, longitud del eje principal $(L r)$ en cm y número de ramas laterales primarias 
$(\mathrm{Re})$; c) de la mazorca [longitud y diámetro de mazorca ( $\mathrm{Lm}, \mathrm{Dm}$ ) en $\mathrm{cm}$, diámetro de olote (Do) en cm, número de hileras (Nh) y número de granos por hilera (Gh)] y d) del grano [peso de 1000 granos (Pm) en g].

\section{Análisis estadístico}

Los datos fueron sometidos a análisis de varianza y, en los casos donde se presentaron resultaron diferencias significativas, se aplicó la prueba de comparación de medias de Tukey $(P \leq 0.05)$; así mismo, se obtuvo la matriz de correlaciones de Pearson, utilizada para realizar un análisis de componentes principales (CP), cuyos valores se utilizaron para representar la dispersión de accesiones y razas de maíz en el plano bidimensional formado por dichos componentes. El resultado permitió identificar las accesiones y razas con mayor diversidad morfológica. Enseguida, se hizo una agrupación de accesiones por el método aglomerativo de Ward (Murtagh y Legendre, 2014). Los datos fueron procesados en los programas SAS ${ }^{\circledR}$ Ver. 9.1.3 (SAS Institute, 2002), Statistica Ver. 7 y Minitab Ver. 19.2.0.

\section{RESULTADOS}

\section{Análisis de varianza}

El análisis de varianza detectó variación significativa $(P \leq 0.01)$ entre las accesiones de maíz nativo para los caracteres agronómicos de planta, espiga, mazorca y grano, lo que prueba la existencia de una diversidad fenotípica importante entre las accesiones y las razas (Cuadro 1). En este mismo cuadro se observan variables con CV menores de 20 \% para Fm, Lp, Lm, Dm, Do, Gh y Pm, así como grados de libertad distintos, lo cual obedece a la variación en el número de accesiones utilizados para cada raza de maíz nativo.

\section{Análisis de correlación}

Los caracteres de planta, espiga y mazorca mostraron un coeficiente $r \geq 0.75(P \leq 0.05)$ con ciertas variables. Valores de correlación positiva fuerte a correlación positiva perfecta $\left(r>0.84^{* *}\right)$ ocurrieron en el crecimiento en longitud de Ap con Am, Lr, Le y Lp; Am con Lr, Le y Lp; Lr con Le y Lp; Le con Lp; así como en Rm con Rg. En cambio, Dm tuvo correlación moderada con Rm y $R g\left(r=0.69^{* *}\right.$ y $r=0.68^{* *}$.

\section{Análisis de componentes principales}

La proporción de la variación total explicada por cada componente principal (CP) se presenta en el Cuadro 2. El CP1 explicó $28.2 \%$ de la variabilidad de los caracteres morfológicos de las poblaciones de maíz nativo, mientras que los CP2, CP3 y CP4 explicaron el 23.4, 10.5 y $6.8 \%$ de la variabilidad, respectivamente. Los cuatro componentes presentan una varianza acumulada de $70 \%$; sin embargo los dos primeros fueron suficientes para explicar de manera aceptable la diversidad de caracteres morfológicos entre accesiones.

La dispersión de accesiones en reveló la integración de éstas en grupos de razas de maíz (Figura 1). En el CP1 destacaron Ver-406 (identificado con la literal $V$ en la Figura 1), Gro-214 (E), Dgo-218 (C) y Nay-191(B) con valores negativos, y Zac-183 (T), Zac-172 (E), SLP-50 y Zac-79 (D) hacia el conjunto de valores positivos, en tanto que en CP2 sobresalieron Ver-406 (V), Mor-99 (P) y Nay-201 (B) en el lado negativo y Jal-633 (E), Gto-101 (C) y Dgo-18 (C) con valores positivos. Por otra parte, en CP3 fueron importantes Jal-333 (C), Jal-19 (C) y Ver-406 (V) y en el CP4 aparecieron SLP-44 (T), Nay-221 (B), Dgo122 (B) y Nay-38 (E). La dispersión de accesiones por cuadrantes formada por CP1 y CP2 (Figura 1) denota que en el Cuadrante II se ubicó el mayor número de accesiones de la misma raza, Celaya (C) con 76 \% (13 accesiones) En el Cuadrante II también se observó $44 \%$ (7) de Elotes Occidentales (E) y, en menor proporción, Harinoso de Ocho (H), Dulce de Jalisco (D), Mushito (M), Pepitilla (P) y Serrano de Jalisco (S, 1 de 2 accesiones). En el Cuadrante III se dispersó 64 \% (7) de accesiones de Bofo, al igual que en los Cuadrantes I (9\%), II (18\%) y IV (9\%); aquí también se localizó 20 \% (2) de accesiones de Pepitilla y las únicas accesiones de Harinoso de Ocho y Zamorano Amarillo.

Los caracteres morfológicos originales medidos en maíces nativos y su contribución a CPs aparecen en el círculo de correlaciones (Figura 2). Los caracteres que aportaron mayor variación al CP1 fueron Le $(r=-0.924)$, Ap $(r=-0.918), \operatorname{Lr}(r=-0.876)$ y $L p(r=-0.843)$, localizados en el Cuadrante III; en cambio, Rm ( $r=0.781)$ y Rg ( $r=$ $0.765)$ en la zona positiva del Cuadrante II aportaron la mayor variación al CP2. En los CP3 y CP4 lo fueron Hi ( $r$ $=0.798)$ y Am $(r=-0.728)$. La sobreposición de imágenes de CP y círculo de correlaciones (Figuras 1 y 2) revela que Ver-406 (V) tuvo caracteres sobresalientes

en Le, $A p$, $L r$ y Lp definidos en el CP1; en la misma situación se colocó a Nay-214 (H), Dgo-218 (C) y Nay-191, Nay-196 y Nay-217 (Bofo) en el Cuadrante III. Por otro lado, en CP2 la mayor variación de Rm y Rg ocurrió en Jal-633 (E), Gto-101 (C) y Dgo-218 (C) en el Cuadrante II, seguidos por Do y Dm; Fm, Lp, Lr, Re y Hm en Ver-406 (V), Mor-99 (P) y Nay-201 (B) en el Cuadrante III.

\section{Análisis de conglomerados}

La continuidad de agrupamientos originó los 
conglomerados que formaron el dendrograma (Figura 3). Los caracteres que aportaron mayor diversidad fueron Le, $A p, L r, L p, R m$ y Rg, con $r>0.75$. El dendrograma revela cinco grupos de accesiones de maíz por su similitud. El Grupo 1 presentó mayor Rg experimental, encabezado por la raza Celaya, que comparte el Subgrupo 1 de las razas Bofo y Elotes Occidentales, mientras que Tabloncillo tuvo menor respuesta productiva en el Subgrupo 2. El Grupo II estuvo integrado por Elotes Occidentales, Pepitilla y Celaya como predominantes. El dendrograma definió dos subgrupos; el Subgrupo 1 integrado por ciertas accesiones de la raza Celaya, en tanto que en el Subgrupo 2 predominó
Celaya junto con Elotes Occidentales y Pepitilla.

El Grupo II se caracterizó por incidir en ciertos caracteres de planta, mazorca y grano. Dos tercios de las accesiones de maíz del Grupo III correspondieron a Bofo, que formó el subgrupo I, y el tercio restante fueron Elotes Occidentales, Zamorano Amarillo y Coscomatepec (Figura 3).

Coscomatepec mostró valores excepcionales en caracteres de planta, mazorca y espiga; Zamorano Amarillo, en cambio, lo fue sólo en caracteres de la mazorca. Con predominio de la raza Mushito, las razas Pepitilla, Bofo

Cuadro 1. Cuadrados medios del análisis de varianza y otros parámetros para 12 razas de maíz nativo evaluadas en Celaya, Guanajuato, México.

\begin{tabular}{|c|c|c|c|c|c|c|}
\hline Variable & & $\begin{array}{l}\text { Grados de } \\
\text { libertad }\end{array}$ & $\begin{array}{l}\text { Cuadrado } \\
\text { medio }\end{array}$ & Error & Media & $\begin{array}{l}\text { Coeficiente de } \\
\text { variación (\%) }\end{array}$ \\
\hline \multicolumn{7}{|l|}{ Caracteres agronómicos } \\
\hline Producción de mazorca (Rm) & & 11 & $18.7 * \star$ & 5.7 & 4.3 t ha $^{-1}$ & 25.3 \\
\hline Producción de grano (Rg) & & 11 & $16.3^{* *}$ & 4.8 & 3.5 t ha $^{-1}$ & 30.9 \\
\hline Producción de olote (Ro) & & 11 & $16.3^{\text {ns }}$ & 0.2 & 0.8 t ha $^{-1}$ & 25.4 \\
\hline Días a floración masculina (Fm) & & 11 & $1345.2 * *$ & 305.3 & $81.1 \mathrm{~d}$ & 20.0 \\
\hline Altura de planta (Ap) & & 9 & $6704.4 * \star$ & 2387 & $170.6 \mathrm{~cm}$ & 28.6 \\
\hline Altura de mazorca (Am) & & 9 & $8301.0 * \star$ & 2618.6 & $124.0 \mathrm{~cm}$ & 41.2 \\
\hline No. Hojas sobre la mazorca (Hm) & & 9 & $2.0 * \star$ & 4.7 & 4.7 & 46.1 \\
\hline \multicolumn{7}{|l|}{ Caracteres de la espiga } \\
\hline Longitud de pedúnculo (Lp) & & 9 & $7176.5 * *$ & 2488.4 & $21.8 \mathrm{~cm}$ & 20.0 \\
\hline $\begin{array}{l}\text { Longitud del tramo del eje con } \\
\text { ramas (Le) }\end{array}$ & & 9 & $8096.8 * *$ & 2598.3 & $22.5 \mathrm{~cm}$ & 22.6 \\
\hline Longitud del eje principal (Lr) & & 9 & $12746.9 *$ & 3876.4 & $24.0 \mathrm{~cm}$ & 25.9 \\
\hline $\begin{array}{l}\text { No. Ramas laterales } \\
\text { primarias }(\mathrm{Re})\end{array}$ & & 9 & $32.6 * *$ & 25.3 & 13.1 & 38.4 \\
\hline \multicolumn{7}{|l|}{ Caracteres de la mazorca } \\
\hline Longitud de mazorca (Lm) & & 10 & $44.5^{\mathrm{ns}}$ & 6.3 & $15.7 \mathrm{~cm}$ & 16 \\
\hline Diámetro de mazorca (Dm) & & 10 & $2.0 * *$ & 0.1 & $4.0 \mathrm{~cm}$ & 7.9 \\
\hline Diámetro de olote (Do) & & 10 & $0.8 * \star$ & 0.1 & $2.7 \mathrm{~cm}$ & 11.7 \\
\hline No. Hileras por mazorca (Hi) & & 10 & $71.5 * \star$ & 14.2 & 11.5 & 32.8 \\
\hline No. Granos por hilera (Gh) & & 10 & $200.1 * \star$ & 18.8 & 25.8 & 16.8 \\
\hline \multicolumn{7}{|l|}{ Caracteres del grano } \\
\hline Peso de mil semillas (Pm) & & 10 & $49082.5 \star \star$ & 2665.1 & $352.9 \mathrm{~g}$ & 14.6 \\
\hline \multicolumn{5}{|c|}{ * $\mathrm{y}$ **: sigificancia estadística con $\mathrm{P} \leq 0.01$ y P $\leq 0.05$, respectivamente, ns: no significativo. } & & \\
\hline Caracter & $\mathrm{CP} 1$ & & $\mathrm{CP} 2$ & CP3 & & $\mathrm{CP} 4$ \\
\hline Valor propio & 4.79 & & 3.97 & 1.78 & & 1.13 \\
\hline Varianza explicada & 28.2 & & 23.4 & 10.5 & & 6.68 \\
\hline Varianza acumulada & 28.22 & & 51.61 & 62.11 & & 68.79 \\
\hline
\end{tabular}




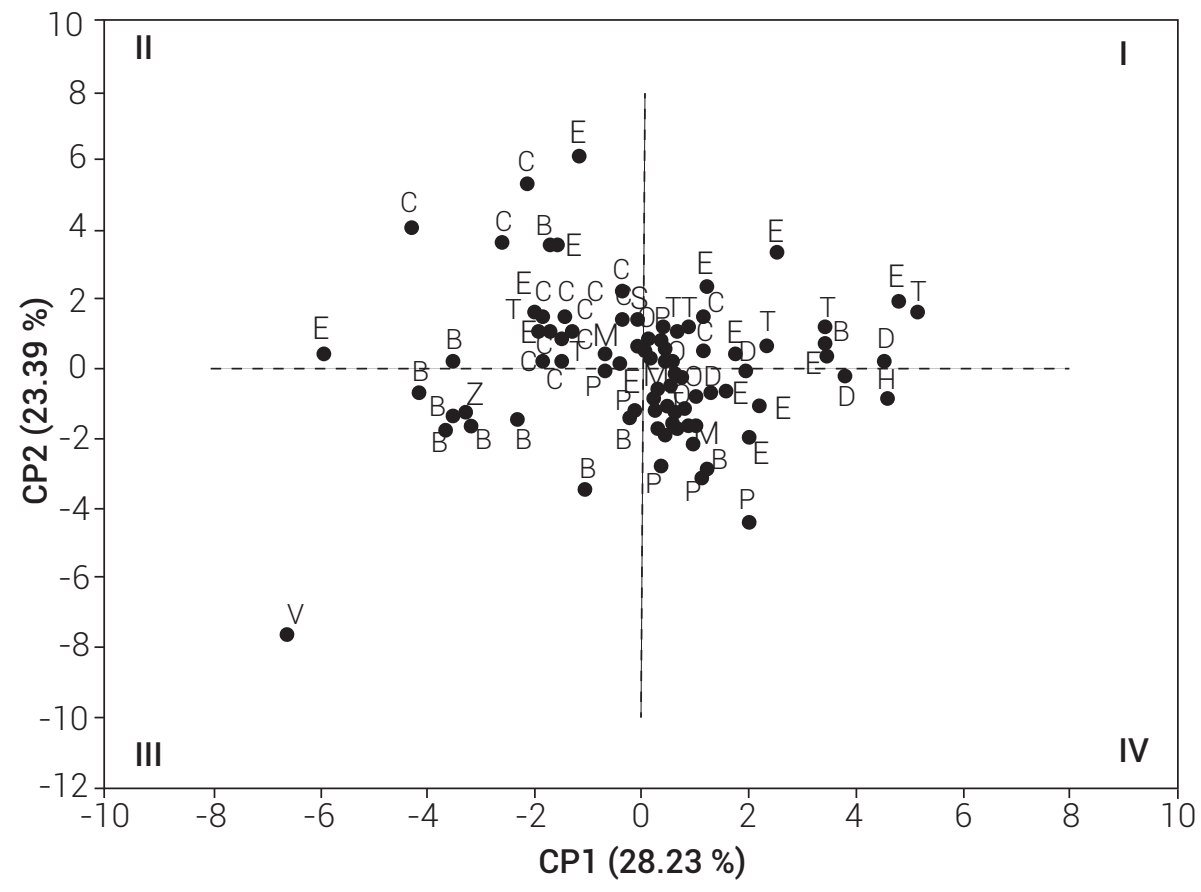

Figura 1. Proyección bidimensional de 90 accesiones de maíz nativo evaluadas en Celaya, Guanajuato, México. Razas Bofo (B), Celaya (C), Coscomatepec (V), Dulce de Jalisco (D), Elotes Occidentales (E), Mushito (M), Pepitilla (P), Serrano de Jalisco (S), Tablilla de Ocho (O), Taboncillo (T) y Zamorano Amarillo (Z). CP1: Componente principal 1, CP2: Componente principal 2.

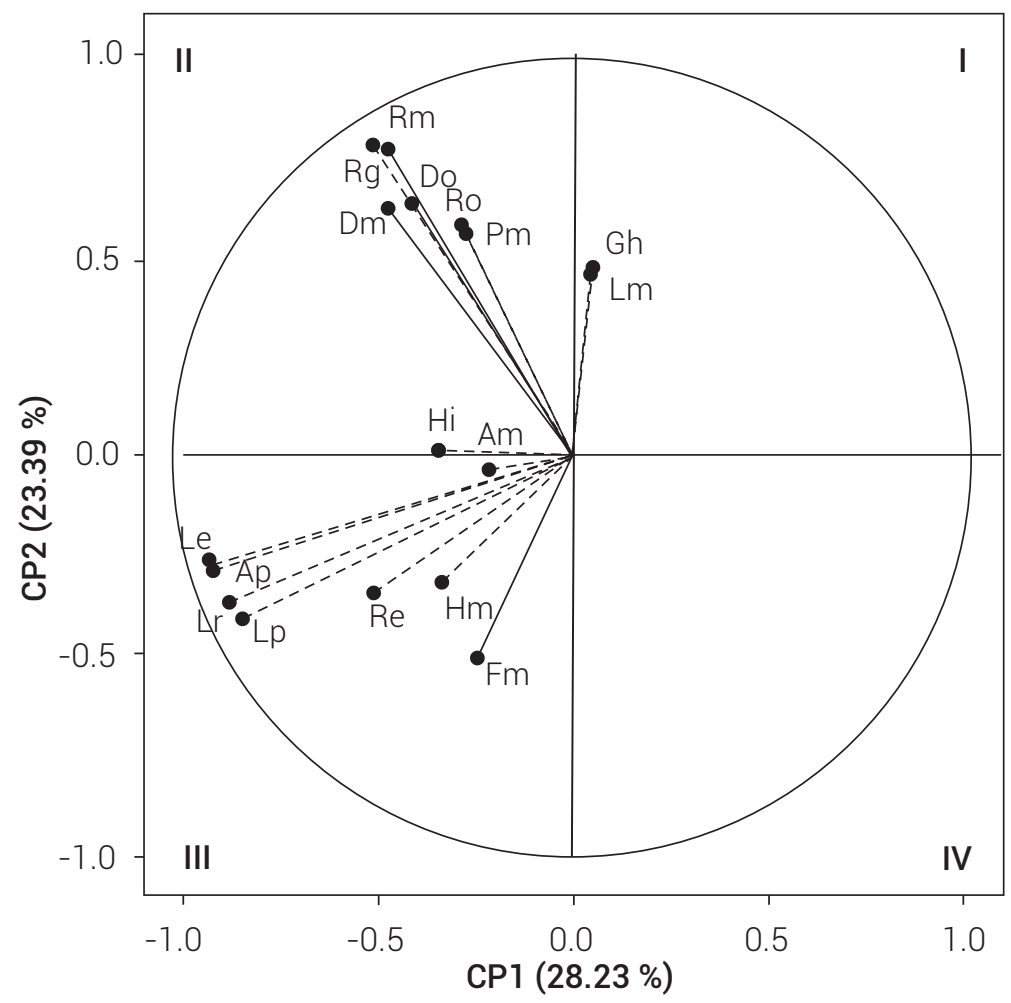

Figura 2. Círculo de correlaciones que muestra la dispersión de caracteres morfológicos de 90 accesiones de 12 razas de maíz nativo en los componentes principales. 


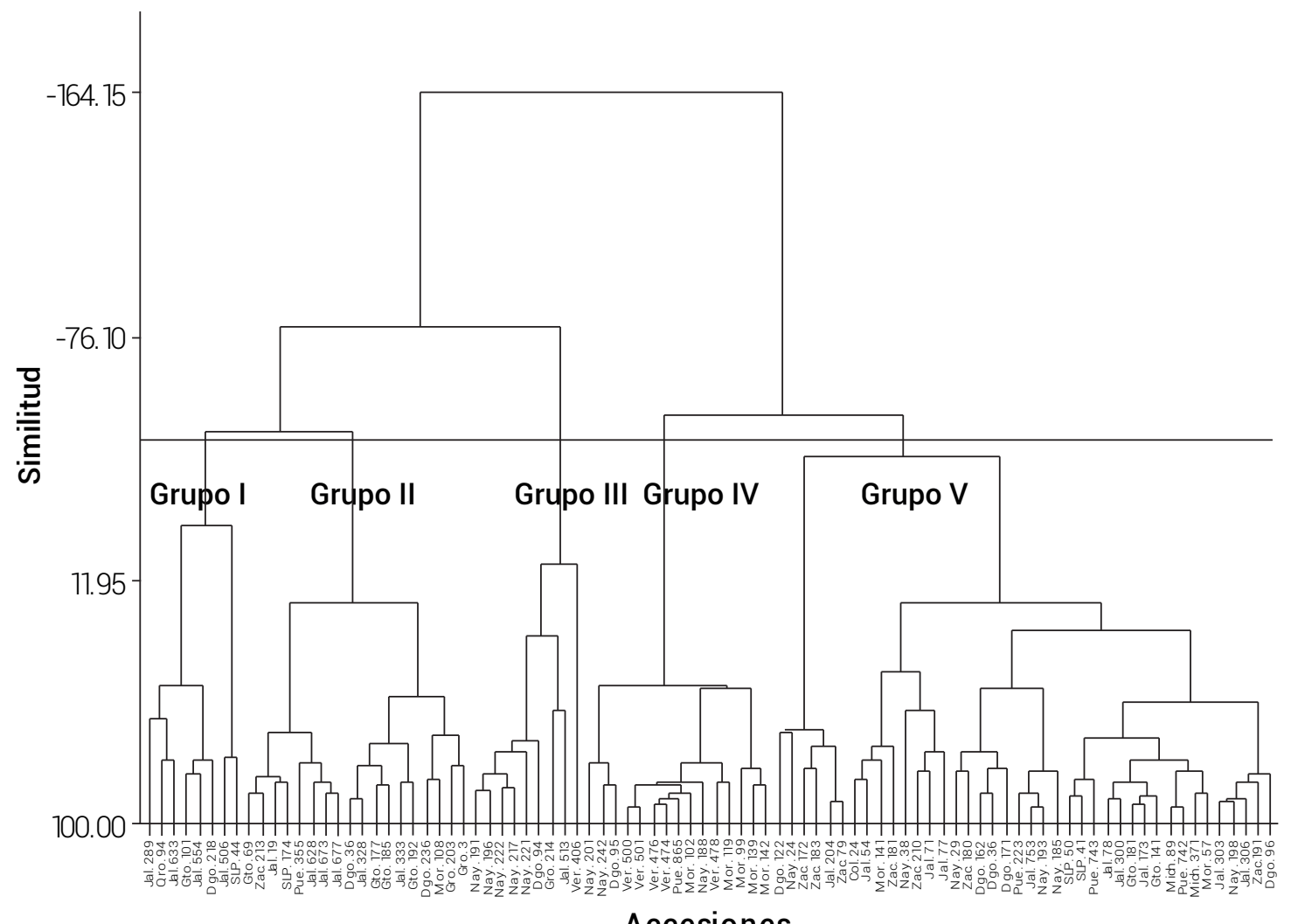

Figura 3. Dendrograma de 90 accesiones de 12 razas de maíz nativo de México, construido mediante el método Ward con distancias euclidianas provenientes de 17 variables morfológicas.

y una sola accesión de Tabloncillo sobresalieron en el Grupo IV del dendrograma (Figura 3), con mayor influencia de caracteres agronómicos de la planta y menor de la mazorca. Cinco subgrupos se formaron en el Grupo IV. El Grupo V fue abundante y diverso en razas; escapan Celaya, Coscomatepec y Zamorano Amarillo. En número de razas del Grupo V, las accesiones de Elotes Occidentales (11) fueron en mayor número que las de Tablilla de Ocho (7), Dulce de Jalisco (6), Tabloncillo (5), Mushito (4), Pepitilla (3), Serrano de Jalisco (2), Bofo (1) y Harinoso de Ocho (1). Así, el Subgrupo 1 fue más variado con cinco razas; la raza Elotes Occidentales dominó en el Subgrupo 2, junto con Dulce de Jalisco y Pepitilla; en el Subgrupo 3 aparecieron accesiones de Tabloncillo, Elotes Occidentales, Tablilla de Ocho, Pepitilla y Serrano de Jalisco. Dulce de Jalisco y Mushito encabezaron el Subgrupo 4, junto con algunas accesiones de Elotes Occidentales, Tabloncillo, Mushito y Serrano de Jalisco, mientras que Tablilla de Ocho fue única en el Subgrupo 5.

\section{DISCUSIÓN}

La variación de los caracteres de las 12 razas de maíz nativo (Cuadro 1) sería atribuida al origen, grado de domesticación o adaptación ambiental y a la amplia gama de genotipos evaluados, por lo que eran de esperarse las diferencias estadísticas en el análisis de varianza. Los valores bajos del coeficiente de variación en Lp y Dm y Hi podrían indicar alta estabilidad ambiental de las razas de maíz implicadas. Al respecto, Muñoz (2005) señaló que la variación genética de maíz es producto del cultivo y selección practicada por agricultores, en respuesta al ambiente y uso antropocéntrico de la especie. El origen genético y geográfico de la raza Bofo (Ortega et al., 2013a) pudo motivar que Harinoso de Ocho y Tabloncillo fueran estadísticamente iguales en Rm (4.8 y $5.5 \mathrm{t} \mathrm{ha}^{-1}$ ) y Rg (3.9 y $\left.3.6 \mathrm{t} \mathrm{ha}^{-1}\right)$; en situación estadística similar estuvo $\mathrm{Rm}$ de Pepitilla (3.3 $\mathrm{t} \mathrm{ha}^{-1}$ ). Salvo Ro en Dulce de Jalisco, Rm y Fm fueron similares en Bofo, Celaya, Elotes Occidentales, Dulce de Jalisco, Mushito, Pepitilla y Tablilla de Ocho; destaca la raza Celaya por menor tiempo de Fm (71 d) y su contraste, Coscomatepec (100 d), cultivada entre 1300 y 2000 msnm en la zona de barlovento y alta nubosidad de la Sierra Madre Oriental en Veracruz y Sierra Norte de Puebla (Ortega et al., 2013a). También Ap, Am, Hm, Lp, Lr y Re fueron significativamente iguales en Bofo, Elotes Occidentales, Dulce de Jalisco, Tabloncillo y Celaya (excepto Lr en esta última). Las razas Celaya, Elotes Occidentales y Pepitilla mostraron significancia estadística en Lp, Re, Lm, Dm, Hi, Gh y Pm (Cuadro 1). Rm fue mayor 
en Gto-101, Dgo-218 y Jal-554 con 9.0, 8.2 y 7.5 tha $^{-1}$ de la raza Celaya y, como consecuencia, Rg fue de $8.3,7.2$ y 6.8 $\mathrm{t} \mathrm{ha}^{-1}$ en cada una, le siguieron Bofo y Elotes Occidentales. Este tipo de respuesta proviene de la evolución del maíz que ha permitido a Celaya, Bofo y Elotes Occidentales adaptarse a ambientes con precipitación pluvial anual menor de 580 mm o cultivarse bajo riego (Ruiz et al., 2008). La raza Celaya se distinguió por el mayor peso de mazorca, y peso y volumen de grano por mazorca (CONABIO, 2020).

La longitud de pedúnculo $(25.3 \mathrm{~cm})$ como caracter de la espiga pudo contribuir al mayor Rg de Dgo-218 de la raza Celaya; sin embargo, no fue estadísticamente comparable con el máximo valor $(40.6 \mathrm{~cm})$ observado en Ver-406 de Coscomatepec, la que expresó mayor Ap (3.73 m) como respuesta probable a su condición genética, en primer lugar, y a la mayor calidad de iluminación recibida, como segunda explicación. El crecimiento óptimo de Coscomatepec ocurre en Chiapas, Oaxaca, Durango, Chihuahua, México, Guerrero, Hidalgo y Querétaro (Ruíz et al., 2013b). En el mismo sentido, algunas accesiones de Bofo y Elotes Occidentales rebasaron los $3.0 \mathrm{~m}$ de altura. En un trabajo afín, 11 accesiones de las razas Clavito, Gallina, Tusilla y Yunga colectadas en la zona Andina de Ecuador mostraron mayor aptitud agronómica en altitudes bajas, lo que supone no sólo adaptación local, sino un proceso evolutivo forzado hacia un ambiente distinto (Tapia et al., 2015).

Un número importante de accesiones alcanzó Fm antes de 70 d, aunque Dgo-36 de la raza Celaya fue más precoz (58 d). Junto a Celaya aparecieron Tablilla de Ocho y Elotes Occidentales, al invertir $63 \mathrm{~d}$ o menos en Fm; en cambio, la única accesión de Coscomatepec y Mor142, Mor-119 y Mor-141 de Pepitilla fueron más tardías (> 100 d). El fotoperíodo en el Bajío de México se amplía desde mitad de año hasta agosto (González et al., 2008), período que coincide con la floración de diversas especies agrícolas, incluyendo maíz. La totalidad de accesiones de Bofo tuvo número alto de $\mathrm{Hm}$. El número de hojas fue de nueve a 11, según la colecta, pero la mayor proporción se tuvo en accesiones provenientes de Nayarit, seguido por Durango y Jalisco; en cambio, $37 \%$ de las accesiones de Elotes Occidentales sumó cuatro hojas sobre la mazorca; la proporción restante de accesiones formó entre cinco y seis hojas. Los granos en $\mathrm{Hi}$ (18) fueron representados en Ver-406, mientras que la mayor proporción de Gh se tuvo en mazorcas de Tablilla de Ocho, Serrano de Jalisco, Tabloncillo, Pepitilla y Elotes Occidentales. En esta última raza sobresalió Dgo-236 con 35 granos por hilera. Por otro lado, Jal-506, Jal-633 y Jal-289 de Tabloncillo, Elotes Occidentales y Bofo tuvieron mayor Pm: 502, 547 y $567 \mathrm{~g}$, respectivamente.
En el análisis de componentes principales la mayoría de los caracteres de planta y espiga fueron expresados en los Cuadrantes II y III (Figura 1), en correspondencia con los grupos raciales de maíz nativo de Bofo, Celaya y Elotes Occidentales, dispersos en el gráfico biplot de CP (Figura 2), una vez sobrepuestas las Figuras 1 y 2 . En el Cuadrante II de éstas se identificó mayor Rm y Rg de Gto101 (9 y $8.3 \mathrm{t} \mathrm{ha}^{-1}$ ), Dgo-18 (8.2 y $7.2 \mathrm{t} \mathrm{ha}^{-1}$ ) y Jal-554 (7.5 y $6.8 \mathrm{t} \mathrm{ha}^{-1}$ ) de la raza Celaya, y Jal-633 (7.4 y $6.0 \mathrm{t} \mathrm{ha}^{-1}$ ) de Elotes Occidentales. Por producción de grano, Celaya es una raza importante en altitudes intermedias, de 1000 a 1800 msnm (CONABIO, 2020). En general, las accesiones de Celaya produjeron de 4.0 a $9.0 \mathrm{t} \mathrm{ha}^{-1}$ de mazorca y de 3.2 a 8.3 t ha $^{-1}$ de grano. Esta amplitud de diferencias de rendimientos explica en parte la diversidad de respuestas entre y dentro de las razas de maíz en la región Bajío, uno de los ambientes más propicios para la actividad agrícola en México y el mundo, en particular para hortalizas y cereales.

Lobo et al. (2003) afirmaron que la variación genética coincide con el ambiente de producción, por la respuesta del germoplasma a factores de crecimiento de la planta. En Celaya, Guanajuato el rendimiento de grano promedio obtenido en temporada de lluvias entre 2000 y 2005 fue de $2 \mathrm{t} \mathrm{ha}^{-1}$ en $76 \%$ de la superficie (correspondiente a temporal) y en riego fue $6.47 \mathrm{t} \mathrm{ha}^{-1}$ (FIRA, 2007). En contraste, Ángeles-Gaspar et al. (2010) registraron de 4.5 a $5.6 \mathrm{t} \mathrm{ha}^{-1}$ de grano en tres poblaciones locales de maíz de Molcaxac, Puebla. México, con lo que superaron a variedades comerciales y confirmaron la mejor adaptación del maíz nativo en la zona, misma situación que se observó en el Istmo de Tehuantepec, Oaxaca (López et al., 2005).

La reducción de Ap y Lp con frecuencia es deseable en el cruzamiento genético (Oliboni et al., 2012). Los caracteres Ap, Le, Lry Retambién contribuyeron a la variación explicada por CP2 para Bofo, predominante en el Grupo III, a través de Nay-196 (Le $=27.2 \mathrm{~cm}$ y $L r=28.9 \mathrm{~cm}$ ) y Nay-191 (Le $=27 \mathrm{~cm}$ y $L r=28.3 \mathrm{~cm}$ ) en el Cuadrante III, y Dgo-94 (Le = $26.9 \mathrm{~cm}$ y $\mathrm{Lr}=28.3 \mathrm{~cm}$ ) en el Cuadrante II, junto a ciertas accesiones de Pepitilla. El resto de las accesiones de Bofo estuvieron dispersas en los Cuadrantes I, II y IV con menor contribución a CP2. La raza Bofo ha sido clasificada como nueva (Ortega et al., 2013a) y pertenece al Grupo de Ocho Carreras, con mejor adaptación a condiciones tropicales y subtropicales (Ruíz et al., 2013a).

En el dendrograma fueron identificados cinco grupos de razas de maíz nativo con similitud. En el Grupo I el mayor Rg fue para Gto-101 y Dgo-218 de Celaya, con 8.3 y $7.2 \mathrm{t}$ ha $^{-1}$. En cambio, Jal-506 y SLP-44 de Tabloncillo tuvieron menor respuesta productiva en el Subgrupo 2, con 3.7 y 4.3 t ha-1 de grano. En general, el Grupo I destacó por mostrar 
mazorcas largas $(17.3 \mathrm{~cm})$ y diámetro grande $(4.7 \mathrm{~cm})$, al igual que Do grueso $(3.1 \mathrm{~cm})$, mayor $\mathrm{Hi}(27.5)$ y Pm $(470$ g). Estos caracteres pudieron definir la mejor respuesta del Grupo I a Rg. La raza Celaya tiene preferencia para mejora genética en el mundo (Ruiz et al., 2013a). Las accesiones Jal-289 (raza B) y Qro-94 y Jal-633 (E) complementaron el Grupo I; la accesión Jal-289 (B) alcanzó mayor Pm (567 g). El Grupo II se caracterizó en lo colectivo por mayor Hi (13) y precocidad de Fm (71.2 d); en lo individual, las accesiones Jal-19 y Jal-333 formaron 16 hileras en la mazorca y Dgo36 tuvo Fm de 58 d, todas de Celaya. El Grupo II también formó granos con menor Pm (241 g) en Dgo-236 (E); el Grupo II también mostró en lo colectivo plantas con Hm bajo (5.0) y, en lo individual, Jal-333 presentó menor Re (9). En el Grupo II, 78 \% de las accesiones perteneció a la raza Celaya; el $12 \%$ restante estuvo dividido por igual entre Elotes Occidentales y Pepitilla. La raza Harinoso de Ocho dio origen a las razas Bofo y Elotes Occidentales (Ortega et al., 2013a); esta última fue probada en una elevación similar a su origen, Nayarit y Altiplanicie de Jalisco, México, bajo ciertas características ecológicas que le hayan sido favorables (Ruíz et al., 2013a). Harinoso de Ocho también dio origen, indirectamente, a la raza Celaya (Hernández y Alanís, 1970).

Dos tercios de las razas de maíz del Grupo III correspondieron a Bofo, que formó el Subgrupo I; el otro tercio fueron Elotes Occidenatles, Zamorano Amarillo y Coscomatepec (Figura 3). Estas dos últimas razas fueron representadas por una accesión cada una. Coscomatepec mostró valores excepcionales en Fm (100 d), Ap (3.7 m), Am $(2.41 \mathrm{~m}), \mathrm{Lp}(40.1 \mathrm{~cm})$, Le $(30.5 \mathrm{~cm})$ y Lr $(34.4 \mathrm{~cm})$, así como Hi (18); Zamorano Amarillo, en cambio, lo fue en Lm $(17 \mathrm{~cm})$ y Dm $(4.9 \mathrm{~cm})$. A la raza Bofo se le localiza desde condiciones subtropicales a tropicales, en elevaciones de 2000 msnm, con requerimiento térmico de 1133 grados día desarrollo (GDD) por ciclo (Ruiz et al., 2013b); en cambio, Coscomatepec es sensible a fotoperíodo y temperatura (Stevenson y Goodman, 1972), que destacan en el Bajío de México. La Fm tardía de Coscomatepece respondería a la presión selectiva de la domesticación, migración y adaptación (Yan et al., 2011). Con predominio de Mushito (40 \%), Pepitilla, Bofo y una sola accesión de Tabloncillo, sobresalieron en el Grupo IV del dendrograma (Figura 3) con Fm amplio (84 a 100 d), mejor representado por Mor142 y Mor-119 de Pepitilla. Cinco subgrupos formaron al Grupo IV. La raza Mushito proviene de zonas altas del centro de México, mientras que Celaya y Pepitilla pertenecen al grupo Dentados Tropicales de alturas medias (Ortega et al., 2013b).

El Grupo $V$ es abundante en número y diversidad de razas (9); escapan Celaya, Coscomatepec y Zamorano Amarillo; en este grupo, el número de accesiones de
Elotes Occidentales $(27.5 \%)$ fue alto. Precisamente, el Subgrupo 1 del Grupo $V$ fue de cinco razas, mientras que Elotes Occidentales sobresalió en el Subgrupo 2, junto con Dulce de Jalisco y Pepitilla. En el Subgrupo 3 sobresalieron accesiones de Tabloncillo, además de Elotes Occidentales, Tablilla de Ocho, Pepitilla y Serrano de Jalisco. Dulce de Jalisco y Mushito encabezaron el Subgrupo 4, con presencia adicional de accesiones de Elotes Occidentales, Tabloncillo, Mushito y Serrano de Jalisco. Tablilla de Ocho fue única en el Subgrupo 5. Los caracteres Ap, Am, Lp, Le, $L r$, Re y Hi fueron bajos entre los grupos de accesiones en el dendrograma.

Los resultados de caracteres de planta, espiga y mazorca difieren de trabajos previos con propósitos similares (Diego-Flores et al., 2012; Guillén-de la Cruz et al., 2014; Hortelano et al., 2012; Rocandio-Rodríguez et al., 2014). En este contexto, algunas de las razas de maíz nativo propuestas en el presente estudio son prometedoras en la región Bajío de México para programas de mejoramiento genético o en cultivos comerciales, de manera que las características fenológicas y morfológicas encontradas en este estudio confirman el patrón biodiverso y variable de las poblaciones de maíz nativo de México en la región Bajío, por lo que tienen potencial para emplearse como portadoras de características individuales en la mejora de líneas y poblaciones futuras.

\section{CONCLUSIONES}

El rendimiento de mazorca y de grano, altura de planta, longitud del tramo del eje con ramas, longitud del eje principal y longitud del pedúnculo, así como días a floración masculina, fueron los caracteres con mayor aportación a la variabilidad fenotípica de los maíces nativos evaluados. Las razas de maíz sobresalientes en producción de grano para el ambiente del Bajío de México fueron Celaya, Elotes Occidentales y Bofo, mientras que Pepitilla, Bofo y Coscomatepec lo fueron en caracteres relacionados con la espiga y fenología. Entre las razas con mayor consistencia aparecen Celaya, Bofo y Elotes Occidentales; en cambio, se restringe la selección de Coscomatepec, Harinoso de Ocho y Zamorano Amarillo probablemente por el bajo número de accesiones probadas.

\section{BIBLIOGRAFÍA}

Ángeles-Gaspar E., E. Ortíz-Torres, P. A. López y G. López-Romero (2010) Caracterización y rendimiento de poblaciones de maíz nativas de Molcaxac, Puebla. Revista Fitotecnia Mexicana 33:287-296, https://doi.org/10.35196/rfm.2010.4.287

Cabrera-Toledo J. M., A. Carballo-Carballo, J. A. Mejía-Contreras, G. GarcíaDe los Santos y H. Vaquera-Huerta (2019) Caracterización de poblaciones sobresalientes de maíz de la raza Zapalote Chico. Revista Fitotecnia Mexicana 42:269-279, https://doi. org/10.35196/rfm.2019.3.269-279 
Chávez-Servia, J. L., P. Diego-Flores y J .C. Carrillo-Rodríguez (2011) Complejos raciales de poblaciones de maíz evaluadas en San Martín Huamelulpán, Oaxaca. Ra Ximhai 7:107-115, https://doi org/10.35197/rx.07.01.2011.10.jc

CONABIO, Comisión Nacional para el Conocimiento y Uso de la Biodiversidad (2020) Razas de maíz de México. Comisión Nacional para el Conocimiento y Uso de la Biodiversidad. Ciudad de México. https://www.biodiversidad.gob.mx/diversidad/alimentos/ maices/razas-de-maiz (Mayo 2021)

CONABIO-TEEB, Comisión Nacional para el Conocimiento y Uso de la Biodiversidad-The Economics of Ecosystems \& Biodiversity (2017) Ecosistemas y agrobiodiversidad en sistemas de producción de maíz de pequeña y gran escala. Comisión Nacional para el Conocimiento y Uso de la Biodiversidad. Ciudad de México. https://www.biodiversidad.gob.mx/media/1/genes/files/ resumen_ejecutivo_TEEB.pdf (Enero 2021).

Coutiño-Estrada B., V. A. Vidal-Martínez y Grisel Sánchez Grajalez (2010) Selección de maíces criollos con calidad elotera bajo condiciones de riego y temporal en Chiapas. In: Mejoramiento, Conservación y Uso de los Maíces Criollos. M. B. Nájera R. y C. A. Ramírez M. (eds.). Publicación Especial. Sociedad Mexicana de Fitogenética. Universidad Michoacana de San Nicolás de Hidalgo, Consejo Estatal de Ciencia y Tecnología del Estado de Michoacán. México. D. F. pp:182-194.

Diego-Flores P., J. C. Carrillo-Rodríguez, J. L. Chávez-Servia y F. CastilloGonzález (2012) Variabilidad en poblaciones de maíz nativo de la Mixteca Baja Oaxaqueña, México. Revista FCA UNCUYO 44:157-171

FIRA, Fideicomisos Instituidos en Relación con la Agricultura (2007) Cultivo de maíz blanco. Análisis de Rentabilidad P-V 2006 y Costos de Cultivo 2007. Residencia Estatal Guanajuato. León, Guanajuato. 44 p. https://www.fira.gob.mx/Nd/MAIZ_PV_Guanajuato_-Rentabilidad_2006_Costos_2007.pdf (Mayo 2020).

García E. (1988) Modificaciones al Sistema de Clasificación Climática de Köppen. Instituto de Geografía, Universidad Nacional Autónoma de México México, D. F 220 p.

González T. G., F. M. Mendoza H., J. Covarrubias P., N. Morán V. y J. A. Acosta G. (2008) Rendimiento y calidad de semilla de frijol en dos épocas de siembra en la región del Bajío. Agricultura Técnica en México 34:421-430

Guillén-de la Cruz P., E. de la Cruz-Lázaro, S. A. Rodríguez-Herrera, G. Castañón-Nájera, A. Gómez-Vázquez y A. J. Lozano-del Río (2014) Diversidad morfológica de poblaciones de maíces nativos (Zea mays L.) del estado de Tabasco, México. Revista FCA UNCUYO 46:239-247.

Hernández X. E. y G. Alanís F. (1970) Estudio morfológico de cinco nuevas razas de maíz de la Sierra Madre Occidental de México: implicaciones filogenéticas y fitogeográficas. Agrociencia 5:330

Herrera-Cabrera B. E., F. Castillo-González, J. J. Sánchez-González, J. M. Hernández-Casillas, R.A. Ortega-Pazkca y M. Major-Goodman (2004) Diversidad del maíz Chalqueño. Agrociencia 38:191-206.

Hortelano S. R. R., A. Gil M., A. Santacruz V., H. López S., P. A. López y S. Miranda C. (2012) Diversidad fenotípica de maíces nativos del altiplano centro-oriente del estado de Puebla, México. Revista Fitotecnia Mexicana 35:97-109, https://doi.org/10.35196/ rfm.2012.2.97

Lobo B. M., C. M. Torres C., J. R. Fonseca, L. A. M. P. de Melo, R. B. Alves and T. Abadie (2003) Characterization of germplasm according to environmental condictions at the collecting site using GIS: Two case studies from Brazil. Plant Genetic Resources Newsletter 135:1-11

López R. G., A. Santacruz V., A. Muñoz 0., F. Castillo G., L. Córdova T. y H. Vaquera H. (2005) Caracterización morfológica de poblaciones nativas de maíz del Istmo de Tehuantepec, México. Interciencia 30:284-290

Muñoz O. A. (2005) Centli Maíz. Prehistoria e Historia, Diversidad, Potencial, Origen Genético y Geográfico. Colegio de Postgraduados. Montecillo, Estado de México. $210 \mathrm{p}$.

Murtagh F. and P. Legendre (2014) Ward's hierarchical agglomerative clustering method: which algorithms implement ward's criterion? Journal of Clasification 31:274-295, https:// doi:10.1007/s00357-014-9161-z
Oliboni R., M. V. Faria, M. Neumann, G. M. Battistelli, R. G. Tegoni and J. T V. de Resende (2012) Genetic divergence among maize hybrids and correlations with heterosis and combining ability. Acta Scientiarum Agronomy 34:37-44, https://doi.org/10.4025/ actasciagron.v34i1.11979

Ortega C. A., M. J. Guerrero H., R. E. Preciado 0. y 0. Cota A. (2013a) Razas de maíz: relaciones filogenéticas y geográficas. In: Diversidad y Distribución del Maíz Nativo y sus Parientes Silvestres de México. A. Ortega C., M. J. Guerrero H. y R. E. Preciado O. (eds.) Printing Arts México. México, D. F. pp:21-43.

Ortega C. A., M. J. Guerrero H., J. M. Hernández C., R. E. Preciado 0., V. A. Vidal M., N. O. Gómez M., ... y F. Rincón S. (2013b) Diversidad y distribución de los maíces nativos en México. In: Diversidad y Distribución del Maíz Nativo y sus Parientes Silvestres en México. A. Ortega C., M. J. Guerrero H. y R.E. Preciado O. (eds.) Printing Arts México. México, D. F. pp:45-171

Ortega-Villegas M .N., L. Zizumbo-Villarreal, N. Monterroso-Salvatierra y 0 . G. Hernández-Lara (2018) Leyes de semillas y maíz transgénico. Análisis desde la co-producción entre ciencia y regímenes económico-políticos en México. Agricultura, Sociedad y Desarrollo 15:413-442, https://doi.org/10.22231/asyd v15i3.854

Rocandio-Rodríguez M., A. Santacruz-Varela, L. Córdova-Téllez, H. LópezSánchez, F. Castillo-González, R. Lobato-Ortíz, ... y R. OrtegaPaczka (2014) Caracterización morfológica y agronómica de siete razas de maíz de los Valles Altos de México. Revista Fitotecnia Mexicana 37:351-361, https://doi.org/10.35196/ rfm.2014.4.351

Ron P. J., J. J. Sánchez G., A. A. Jiménez C., J. A. Carrera V., J. G. Martín L., M. M. Morales R., ... y J. G. Rodríguez F. (2006) Maíces nativos del occidente de México. I. Colectas de 2004. Scientia-CUCBA 8:1-139.

Ruíz C. J. A., N. Durán P., J. J. Sánchez G., J. Ron P., D. R. González E., J. B. Holland and G. Medina G. (2008) Climatic adaptation and ecological descriptors of 42 Mexican maíze races. Crop Science 48:1502-1512, https://doi.org/10.2135/cropsci2007.09.0518

Ruiz C. J. A., J. M. Hernández C., J. J. Sánchez G., A. Ortega C., G. Ramírez 0. M. J. Guerrero H., ... y L. de la Cruz L. (2013a) Ecología, Adaptación y Distribución Actual y Potencial de las Razas Mexicanas de Maíz. Libro Técnico Núm. 5. Campo Experimental Centro Altos de Jalisco, INIFAP. Tepatitlán de Morelos, Jalisco.159 p.

Ruiz C. J. A., A. Ortega C., G. Ramírez 0., M. J. Guerrero H. y J. J. Sánchez G. (2013b) Distribución actual y potencial de las razas Mexicanas de maíz. In: Diversidad y Distribución del Maíz Nativo y sus Parientes Silvestres en México. A. Ortega C., M. J. Guerrero H. y R. E. Preciado O. (eds.). Printing Arts México. México, D. F. pp:172-208.

SAGARPA-INIFAP-SENASICA, Secretaría de Agricultura, Ganadería, Desarrollo Rural, Pesca y Alimentación-Instituto Nacional de Investigaciones Forestales, Agrícolas y Pecuarias-Servicio Nacional de Sanidad, Inocuidad y Calidad Alimentaria (2015) Maíz punta de riego In: Agenda Técnica Agrícola Guanajuato. Segunda edición. Secretaría de Agricultura, Ganadería, Desarrollo Rural, Pesca y Alimentación. México, D. F. pp:225-232

SAGARPA-SNICS, Secretaría de Agricultura, Ganadería, Desarrollo Rural, Pesca y Alimentación-Servicio Nacional de Inspección y Certificación de Semillas (2014) Maíz (Zea mays L.). Guía Técnica para la Descripción Varietal. Servicio Nacional de Inspección y Certificación de Semillas. Tlalnepantla, Estado de México. 39 p. https://doi.org/10.13140/RG.2.2.12433.22883

SAS Institute (2007) The SAS System for Windows Microsoft. Guide for Personal Computer. SAS/STAT ver. 9.1.3. SAS Institute, Inc Cary, North Carolina, USA. 212 p.

SIAP, Servicio de Información Agroalimentaria y Pesquera (2020) Anuario estadístico de la producción agrícola. Secretaría de Agricultura y Desarrollo Rural. Ciudad de México. https://nube.siap.gob. mx/cierreagricola/ (Mayo 2020).

Stevenson J. C. and M. M. Goodman (1972) Ecology of exotic races of maize. I. Leaf number and tillering of 16 races under four temperatures and two photoperiods. Crop Science 12:864-868, https://doi.org/10.2135/cropsci1972.0011183X00120006004 $5 x$ 
Tapia C., E. Torres and M. Parra-Quijano (2015) Searching for adaptation to abiotic stress: ecogeographical analysis of highland Ecuadorian maize. Crop Science 55:262-274, https://doi.org/10.2135/ cropsci2013.12.0813
Yan J., M. Warburton and J. Crouch (2011) Association mapping for enhancing maize (Zea mays L.) genetic improvement Crop Science 51:433-449, https://doi.org/10.2135/ cropsci2010.04.0233 